\title{
INTELIGENCIA LÓGICO MATEMÁTICO Y RENDIMIENTO ACADÉMICO EN ESTUDIANTES DE LA FACULTAD INGENIERÍA Y CIENCIAS HUMANAS - UNCP
}

\author{
Héctor Basilio M. y Carmen Espinoza T. Emerson Julca M. \\ Facultad de Ingeniería y Ciencias Humanas, Universidad Nacional del Centro del Perú, Perú.
}

\begin{abstract}
RESUMEN
La investigación surge de la interrogante ¿Qué relación existe entre la inteligencia lógico matemático y el rendimiento académico en los estudiantes de la Facultad de Ingeniería y Ciencias Humanas de la UNCP?. El tipo de investigación fué el descriptivo, con un diseño correlacional. Para la medición de la inteligencia lógico matemático se aplicó una prueba pedagógica y para el rendimiento académico se utilizó la base de datos de la Facultad de Ingeniería y Ciencias Humanas de la UNCP semestre 2011-II. El análisis de datos se realizó con la estadística descriptiva, correlacional "r" Pearson e inferencial la prueba "t" de student. La muestra estuvo constituido por 91 estudiantes de la Facultad de Ingeniería y Ciencias Humanas de la UNCP seleccionado con un muestreo probabilístico, concluyendo que la inteligencia lógico matemático, presenta una correlación positiva alta con el rendimiento académico".
\end{abstract}

Palabras clave: Inteligencia, Matemáticas, inteligencia lógico matemática rendimiento académico.

\section{LOGICAL MATHEMATICAL INTELLIGENCE AND ACADEMIC ACHIEVEMENT IN FACULTY OF ENGINEERING AND HUMAN SCIENCES - UNCP}

\begin{abstract}
The research arises from the question What is the relationship between logical mathematical intelligence and academic achievement in students of the Faculty of Engineering and Humanities of the UNCP?. The research was the descriptive, correlational design. For the measurement of logical mathematical intelligence test was applied to teaching and academic performance was used the database of the Faculty of Engineering and Humanities of the half UNCP 2011-II. Data analysis was performed using descriptive statistics, correlation " $r$ " Pearson and inferential proof "t" student. The sample consisted of 91 students from the Faculty of Engineering and Humanities of the UNCP selected with a probability sample, concluding that logical mathematical intelligence, has a high positive correlation with academic performance.
\end{abstract}

Keywords: Intelligence, Mathematics, mathematical logic intelligence and academic performance. 


\section{INTRODUCCIÓN}

La inteligencia lógica se concibe como el uso de la competencia cognitiva para operar con proposiciones, de tal forma que partiendo de lo conocido o de lo que se cree dominar en términos de operación mental el sujeto pueda ir a lo desconocido cuyos elementos sean previsiblemente homólogos 0 equivalentes. Esta capacidad constituye un elemento central para la resolución de problemas, y resulta esencial en el desarrollo y fortalecimiento del razonamiento, la deducción y en general el pensamiento abstracto (Ortega; 2005).

Por otro lado, aunque no sea la única, la matemática emerge como un campo de experimentación privilegiado para el desarrollo del pensamiento lógico; todo ello ha hecho relacionar desde siempre, pensamiento lógico y el rendimiento matemático. Según Gardner (1995), Ios estudiantes que manifiestan un alto nivel en el dominio matemático, disfrutan especialmente con operaciones que involucran números, les atrae enormemente combinarlos y emplear fórmulas para ello y sienten curiosidad y placer por los problemas no resueltos que excitan su curiosidad, explorando y experimentando con ellos.

Se ha encontrado que la inteligencia lógico matemático, presenta una relación directa, estadísticamente significativa y de magnitud moderada con el rendimiento académico general

Frente a lo expuesto, la presente investigación tiene por finalidad determinar la relación que existe entre la inteligencia lógico matemático y el rendimiento académico en los estudiantes de la Facultad de Ingeniería y Ciencias Humanas de la UNCP.

El contenido del estudio se ha organizado en $\mathrm{V}$ capítulos: El primero está referido sobre el planteamiento del problema su caracterización y su formulación, el objetivo general y los específicos; el segundo capítulo presenta el marco teórico, el planteamiento de la hipótesis las variables y su respectiva operacionalización. El tercer capítulo está constituido por la metodología de la investigación, el tipo, nivel y diseño de investigación; el cuarto presenta el trabajo de campo y proceso de contraste de la hipótesis. Y por último el quinto y último capítulo se detalla la discusión de resultados acorde a los antecedentes y la teoría de la investigación. Para luego redactar las conclusiones, recomendaciones, bibliografía y anexos.

En la ejecución del presente trabajo de investigación se ha tenido limitaciones referidos a la parte teórica, de espacio de aplicación y de tiempo de ejecución; los cuales fueron solucionados mediante la revisión de las bases teóricas gracias a la bibliografía especializada de la escuela de post grado de la UNCP; el resto de las limitaciones fueron resueltos gracias a la buena planificación en coordinación con el asesor para la ejecución y aplicación del instrumento de las variables de estudio.

\section{MATERIAL Y MÉTODOS}

\section{Tipo de investigación}

El tipo de investigación es aplicada; Según Sánchez y Reyes (1996), manifiestan que la investigación aplicada se denomina también constructiva 0 utilitaria se caracteriza por su interés en la aplicación de los conocimientos teóricos a una determinada situación; en la presente investigación se construyó y validó la prueba de capacidades de inteligencia lógico matemático en estudiantes de la Facultad de Ingeniería y Ciencias Humanas de Junín.

\section{Nivel de investigación:}

El nivel de investigación correlacional según Hernández y otros (2003) "La investigación correlacional tiene como propósito evaluar la relación que exista entre dos o más variables o conceptos".

En la presente investigación se correlacionó la inteligencia lógico matemático y el rendimiento académico en los estudiantes de la Facultad de Ingeniería y Ciencias Humanas de la UNCP.

\section{Método de investigación:}

Según Sánchez y Reyes (1996), el método es descriptivo porque consiste en describir, analizar e interpretar sistemáticamente hechos relacionados con la variable inteligencia lógico matemático y el rendimiento académico en los estudiantes de la Facultad de Ingeniería y Ciencias Humanas de Junín. Así mismo se utilizó el método inductivo porque al evaluar la inteligencia lógico matemático se partió de datos particulares para así llegar a conclusiones generales. (Carrasco; 2009). 
Así mismo se utilizó el método bibliográfico porque ayudo obtener información, ya sea de fuentes secundarias contenidas en libros, artículos de revistas, publicaciones, investigaciones, internet, etc.

Se utilizó también el método estadístico cuantitativo porque ayudó medir matemáticamente la relación que existe entre la inteligencia lógico matemático y el rendimiento académico en los estudiantes de la Facultad de Ingeniería y Ciencias Humanas y los fenómenos sociales que intervinieron en la investigación. Se partió de la aplicación de un instrumento de psicometría donde se obtuvo datos cuantitativos (Tapia; 1999)

\section{Diseño de la investigación:}

El diseño considerado es el descriptivo correlacional, cuyo esquema es el siguiente:

M

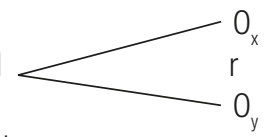

Donde:

M : Muestra de los estudiantes de la Facultad de Ingeniería y Ciencias Humanas.

$0_{x}$ : Observación de la Inteligencia Lógico Matemático

$0_{y}:$ Observación del rendimiento académico

$r$ : Coeficiente de correlación entre ambas variables.

\section{POBLACIÓN Y MUESTRA:}

\section{Población Objetiva:}

Está conformada por los estudiantes de la Universidad Nacional del Centro del Perú.

\section{Población Accesible:}

Conformada por 120 estudiantes de la Facultad de Ingeniería y Ciencias Humanas de la UNCP divido en dos escuelas 46 en la escuela de Ing. Agroindustrial y 74 en la escuela de Enfermería

\section{Muestra:}

Se realizo el muestreo probabilístico donde todos los elementos de la población tuvieron la misma posibilidad de ser escogidos. (Hernández y otros; 2003).
$n^{\prime}=\frac{Z^{2} p \cdot q}{(s e)^{2}}=\frac{1,96^{2}(0,5)(0,5)}{(0,05)^{2}}=384$

$n=\frac{n^{\prime}}{1+n^{\prime} / N}=\frac{384}{1+384 / 120}=91$

Para seleccionar a los 91 estudiantes se utilizo el muestro probabilístico estratificado:

Factor: 91/120

\begin{tabular}{|l|c|c|c|}
\hline \multicolumn{4}{|c|}{ FACULTAD DE INGENIERÍA Y CIENCIAS HUMANAS } \\
\hline Semestre & $\begin{array}{c}\text { EAP: } \\
\text { Ing. Agroindustrial }\end{array}$ & $\begin{array}{c}\text { EAP: } \\
\text { Enfermería }\end{array}$ & Total \\
\hline II Semestre & 11 & 21 & 32 \\
\hline IV Semestre & 6 & 24 & 30 \\
\hline VI Semestre & 6 & 11 & 17 \\
\hline VIII Semestre & 8 & -- & 8 \\
\hline X Semestre & 4 & -- & 4 \\
\hline Total & 35 & 56 & 91 \\
\hline
\end{tabular}

$=0,76$

Fuente: Archivo asuntos académicos de la Facultad

\section{RESULTADOS}

Análisis comparativo entre inteligencia lógico matemático y rendimiento académico

TABLA 07 Correlación: inteligencia lógico matemático y rendimiento académico.

\begin{tabular}{|l|l|l|}
\multicolumn{4}{|c|}{ Correlaciones } \\
\hline & $\begin{array}{c}\text { Rendi- } \\
\text { miento }\end{array}$ & Inteligencia \\
\hline \multirow{2}{*}{$\begin{array}{l}\text { Correlación de Pearson } \\
\text { R Sig. (bilateral) } \\
\text { N }\end{array}$} & 1 &, $736^{* *}$ \\
\hline & Correlación de Pearson \\
Sig. (bilateral) &, $736^{* *}$ & 1 \\
N &, 000 & \\
\hline
\end{tabular}

**. La correlación es significativa al nivel 0,05 (bilateral) Fuente: Archivo del investigador.

En la tabla $N^{\circ} 07$ se muestra la correlación entre las variables de lainvestigación inteligencialógico matemático y rendimiento académico; el coeficiente de correlación $r$ de pesaron es igual 0,736 resulta alta y positiva por 
lo que se puede decir que existe relación directa entre la inteligencia lógico matemático y el rendimiento académico en los estudiantes de la FICCHH - UNCP

GRAFICO 07 Dispersión: Inteligencia lógico matemático - rendimiento académico

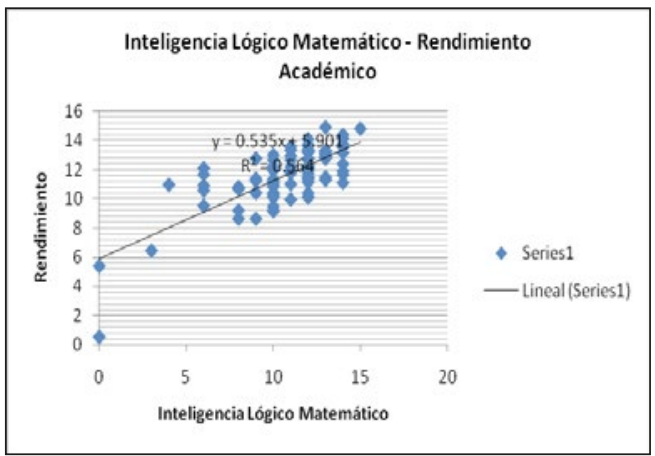

Proceso de la prueba de hipótesis

Para las hipótesis planteadas se aplicó la Prueba "t de Student"

\section{PRUEBA DE LAS HIPÓTESIS ESPECÍFICAS}

\section{HIPÓTESIS ESPECÍFICA 1:}

\section{a) Hipótesis Operacional:}

$\mathbf{H}_{0}$ : No existe una relación directa entre la capacidad de razonamiento y demostración y el rendimiento académico en los estudiantes de la Facultad de Ingeniería y Ciencias Humanas de la UNCP

$$
\mathrm{Ho}: \rho=0
$$

$\mathbf{H}_{\mathbf{1}}$ : Existe una relación directa entre la capacidad de razonamiento y demostración y el rendimiento académico en los estudiantes de la Facultad de Ingeniería y Ciencias Humanas de la UNCP.

$$
H_{1}: \rho \neq 0
$$

\section{b) Nivel de Significancia:}

$\alpha=0.05$, es decir el $5 \%$.

c) Muestra (N):

$\mathrm{N}=91$ d) $r$ de Pearson:

Teniendo:

$$
r=0,671
$$

e) Estadística de prueba:

$t=\frac{r \cdot \sqrt{N-2}}{\sqrt{1-r^{2}}}$

$t=\frac{0,671 \cdot \sqrt{91-2}}{\sqrt{1-(0,671)^{2}}}$

$t_{c}=8,5375$

f) Región de Rechazo - Aceptación y cálculos:

De acuerdo con:

$\alpha=0,05$

$\mathrm{gl}=\mathrm{N}-1=91-1=90$

Encontramos:

Valor crítico $= \pm 1,6620$, tal como se puede apreciar en la Tabla

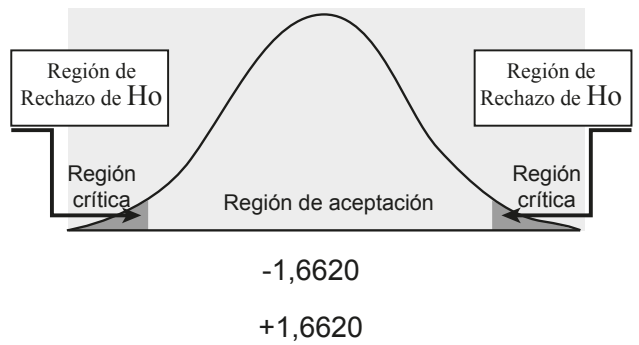

g) Decisión estadística:

Puesto que la t calculada tc $=8,5375$ la cual se encuentra en la zona de rechazo de $\mathrm{Ho}$, por tanto se acepta la hipótesis alterna $\left(\mathrm{H}_{1}\right)$.

h) Conclusión estadística:

Al aceptar la $\mathrm{H}_{1}$, podemos afirmar que existe una relación directa entre la capacidad de razonamiento y demostración y el rendimiento académico en los estudiantes de la Facultad de Ingeniería y Ciencias Humanas de la UNCP. 


\section{HIPÓTESIS ESPECÍFICA 2:}

\section{a) Hipótesis Operacional:}

$\mathbf{H}_{0}$ : No existe una relación directa entre la capacidad de razonamiento deductivo y el rendimiento académico en los estudiantes de la Facultad de Ingeniería y Ciencias Humanas de la UNCP

$$
\mathrm{Ho}_{0}: \rho=0
$$

$\mathbf{H}_{\mathbf{1}}$ : Existe una relación directa entre la capacidad de razonamiento deductivo y el rendimiento académico en los estudiantes de la Facultad de Ingeniería y Ciencias Humanas de la UNCP.

$$
H_{1}: \rho \neq 0
$$

b) Nivel de Significancia: $\alpha=0,05$, es decir el $5 \%$.

c) Muestra (N): $\mathrm{N}=91$

d) $r$ de Pearson: Teniendo: $r=0,367$

e) Estadística de prueba:

$$
\begin{aligned}
& t=\frac{r \cdot \sqrt{N-2}}{\sqrt{1-r^{2}}} \\
& t=\frac{0,367 \cdot \sqrt{91-2}}{\sqrt{1-(0,367)^{2}}} \\
& t_{c}=3,722
\end{aligned}
$$

f) Región de Rechazo - Aceptación y cálculos:

De acuerdo con: $\alpha=0,05$

$\mathrm{gl}=\mathrm{N}-1=91-1=90$

Encontramos:

Valor crítico $= \pm 1,6620$, tal como se puede apreciar en la Tabla

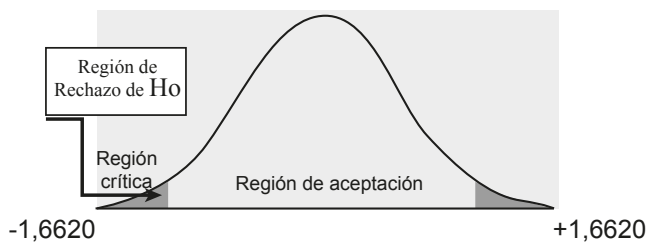

g) Decisión estadística:

Puesto que la t calculada tc $=3,722$ la cual se encuentra en la zona de rechazo de $\mathrm{Ho}$, por tanto se acepta la hipótesis alterna $\left(\mathrm{H}_{1}\right)$.

h) Conclusión estadística:

Al aceptar la $H_{1}$, podemos afirmar que existe una relación directa entre la capacidad de razonamiento deductivo y el rendimiento académico en los estudiantes de la Facultad de Ingeniería y Ciencias Humanas de la UNCP

\section{HIPÓTESIS ESPECÍFICA 3:}

\section{a) Hipótesis Operacional:}

$\mathbf{H}_{\mathbf{0}}$ : No existe una relación directa entre la capacidad de razonamiento inductivo y el rendimiento académico en los estudiantes de la Facultad de Ingeniería y Ciencias Humanas de la UNCP.

$$
\text { Ho }: \rho=0
$$

$\mathbf{H}_{\mathbf{1}}$ : Existe una relación directa entre la capacidad de razonamiento inductivo y el rendimiento académico en los estudiantes de la Facultad de Ingeniería y Ciencias Humanas de la UNCP.

$$
H_{1}: \rho \neq 0
$$

b) Nivel de Significancia: $\alpha=0,05$, es decir el $5 \%$.

\section{c) Muestra (N):}

$$
\mathrm{N}=91
$$

\section{d) $r$ de Pearson:}

Teniendo: $\quad r=0,511$

e) Estadística de prueba:

$$
\begin{aligned}
& t=\frac{r \cdot \sqrt{N-2}}{\sqrt{1-r^{2}}} \\
& t=\frac{0,511 \cdot \sqrt{91-2}}{\sqrt{1-(0,511)^{2}}} \\
& t_{c}=5,6083
\end{aligned}
$$


f) Región de Rechazo - Aceptación y cálculos:

De acuerdo con:

$\alpha=0,05$

$\mathrm{gl}=\mathrm{N}-1=91-1=90$

\section{Encontramos:}

Valor crítico $= \pm 1,6620$, tal como se puede apreciar en la Tabla

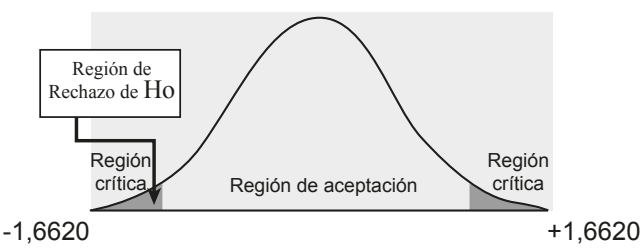

g) Decisión estadística:

Puesto que la t calculada tc $=5,6083$ la cual se encuentra en la zona de rechazo de Ho, por tanto se acepta la hipótesis alterna $\left(\mathrm{H}_{1}\right)$.

h) Conclusión estadística:

Al aceptar la $\mathrm{H}_{1}$, podemos afirmar que existe una relación directa entre la capacidad de razonamiento inductivo y el rendimiento académico en los estudiantes de la Facultad de Ingeniería y Ciencias Humanas de la UNCP

\section{HIPÓTESIS ESPECÍFICA 4:}

a) Hipótesis Operacional:

$\mathbf{H}_{\mathbf{0}}$ : No existe una relación directa entre la capacidad de interpretación de gráficos y figuras y el rendimiento académico en los estudiantes de la Facultad de Ingeniería y Ciencias Humanas de la UNCP.

$$
\mathrm{H}_{0}: \rho=0
$$

$\mathbf{H}_{\mathbf{1}}$ : Existe una relación directa entre la capacidad de interpretación de gráficos y figuras y el rendimiento académico en los estudiantes de la Facultad de Ingeniería y Ciencias Humanas de la UNCP.

$$
H_{1}: \rho \neq 0
$$

\section{b) Nivel de Significancia: \\ $\alpha=0,05$, es decir el $5 \%$.}

\section{C) Muestra (N):}

$\mathrm{N}=91$ d) $r$ de Pearson:

Teniendo: $\quad r=0,380$

e) Estadística de prueba:

$t=\frac{r \cdot \sqrt{N-2}}{\sqrt{1-r^{2}}}$

$t=\frac{0,380 \cdot \sqrt{91-2}}{\sqrt{1-(0,380)^{2}}}$

$t_{c}=3,8756$

f) Región de Rechazo - Aceptación y cálculos:

De acuerdo con:

$\alpha=0,05$

$\mathrm{gl}=\mathrm{N}-1=91-1=90$

Encontramos:

Valor crítico $= \pm 1,6620$, tal como se puede apreciar en la Tabla

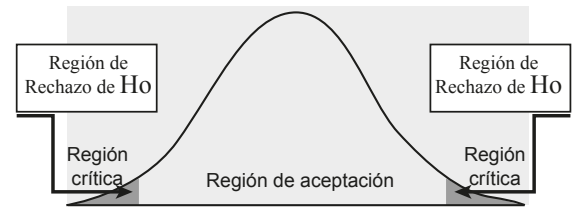

$-1,6620$

$+1,6620$

\section{g) Decisión estadística:}

Puesto que la t calculada tc $=3,8756$ la cual se encuentra en la zona de rechazo de Ho, por tanto se acepta la hipótesis alterna $\left(\mathrm{H}_{1}\right)$.

h) Conclusión estadística:

Al aceptar la $\mathrm{H}_{1}$, podemos afirmar que existe una relación directa entre la capacidad de interpretación de gráficos y figuras y el rendimiento académico en los estudiantes de la Facultad de Ingeniería y Ciencias Humanas de la UNCP 


\section{PRUEBA DE LA HIPÓTESIS GENERAL}

\section{a) Hipótesis Operacional:}

$\mathbf{H}_{\mathbf{0}}$ : No existe una relación directa entre la inteligencia lógico matemático y el rendimiento académico en los estudiantes de la Facultad de Ingeniería y Ciencias Humanas de la UNCP.

$$
\text { Ho }: \rho=0
$$

$\mathbf{H}_{\mathbf{1}}$ : Existe una relación directa entre la inteligencia lógico matemático y el rendimiento académico en los estudiantes de la Facultad de Ingeniería y Ciencias Humanas de la UNCP.

$$
H_{1}: \rho \neq 0
$$

b) Nivel de Significancia: $\alpha=0,05$, es decir el $5 \%$.

c) Muestra (N): N = 91

d) $r$ de Pearson: Teniendo: $\quad r=0,736$

e) Estadística de prueba:

$$
\begin{gathered}
t=\frac{r \cdot \sqrt{N-2}}{\sqrt{1-r^{2}}} \quad t=\frac{0,736 \sqrt{91-2}}{\sqrt{1-(0,736)^{2}}} \\
t_{c}=10,2564
\end{gathered}
$$

\section{f) Región de Rechazo -}

\section{Aceptación y cálculos:}

De acuerdo con:

$\alpha=0,05$

$\mathrm{gl}=\mathrm{N}-1=91-1=90$

Encontramos:

Valor crítico $= \pm 1,6620$, tal como se puede apreciar en la Tabla

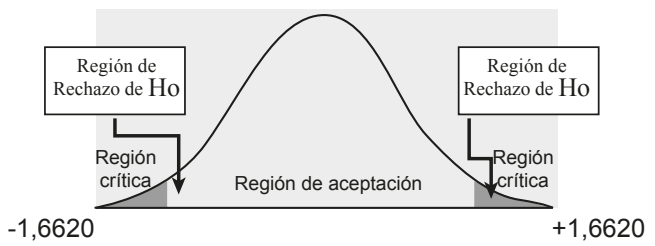

\section{g) Decisión estadística:}

Puesto que la t calculada tc $=10,2564$ la cual se encuentra en la zona de rechazo de $\mathrm{Ho}$, por tanto se acepta la hipótesis alterna $\left(\mathrm{H}_{1}\right)$.

\section{h) Conclusión estadística:}

Al aceptar la $H_{1}$, podemos afirmar que existe una relación directa entre la inteligencia lógico matemático y el rendimiento académico en los estudiantes de la Facultad de Ingeniería y Ciencias Humanas de la UNCP.

\section{DISCUSIÓN}

El análisis multivariado de las variables Inteligencia Lógico Matemático y Rendimiento Académico mediante la prueba del coeficiente de correlación " $r$ " de Pearson arrojó un valor de 0,736 con un nivel de confianza de $95 \%$, el resultado indica que las variables antes mencionadas se relacionan positivamente; de esta manera se confirma lo mencionado por Gardner (1999) donde explica que los individuos especialmente dotados de inteligencia lógico matemático tienen una facilidad en la resolución de problemas y expresan un buen rendimiento en matematicas.

La media en inteligencia lógico matemático alcanzó un puntaje de 10,7363 mientras que la moda y la mediana coincidieron con un puntaje de 12, esto confirma lo mencionado por Antunes (2004) donde refiere que los seres humanos están dotados por inteligencias múltiples; esto quiere decir que un individuo no solo tiene inteligencia lógico matemático si no tiene cualidades para otro tipo de inteligencia.

La media en el rendimiento académico alcanzó un puntaje de 11,58 mientras que la moda tiene un puntaje de 12 y la mediana llego a 13. Lo que se concluye que solo en la moda ambos puntajes son iguales, gracias al trabajo de de Vildoso (2003) podemos concluir que el rendimiento académico universitario es un resultado del aprendizaje suscitado por la actividad del docente, y producido en el estudiante aunque es claro que no todo aprendizaje es producto de la acción del docente pero en la mayoría de los casos se da en forma cualitativa y cuantitativa.

Mediante la prueba $t$ de student, se obtiene un valor $\mathrm{tc}=10,2564>\mathrm{t}$ de la tabla. Se acepta la hipótesis alterna, es decir, existe relación directa entre 
la inteligencia lógico matemático y el rendimiento académico en los estudiantes de la Facultad de Ingeniería y Ciencias Humanas de la UNCP.

\section{CONCLUSIONES}

La variable inteligencia lógico matemático tiene correlación positiva con el Rendimiento Académico en estudiantes de la Facultad de Ingeniería y Ciencias Humanas de la UNCP, lo que implica que a mayor nivel de inteligencia lógico matemático, mayor será el rendimiento académico.

\section{REFERENCIAS BIBLIOGRÁFICAS:}

1. Almeida, Á. (2006). . La intervención de la memoria de trabajo en el aprendizaje del cálculo aritmético. Tesis Doctoral, Universitat Autónoma de Barcelona.

2. Asociación educativa Trilce (2005) Logica Psicología Lima - Perú

3. Asociación de docentes para postulantes a la Universidad Nacional de Ingeniería Aduni (2001) Razonamiento Matemático Lima: Lumbreras

4. Baddeley, A. D. (1998). . Memoria Humana. Teoría y Práctica. Madrid: McGraw-Hill.

5. Baddeley, A. D. (2003). Working memory: looking back and looking forward. En: . Neuroscience, Vol. 4; 829-839.

6. Beltrán, J. (1993). . Procesos, estrategias y técnicas de aprendizaje. Madrid: Editorial Síntesis.

7. Cornelio, A. (2008) . Relación entre la gestión pedagógica y el aprendizaje del área lógico matematico de los alumnos del curto grado de primaria de menores de la I.E. Sor Ana Angeles del callo.

8. Etchepareborda, M.C. y Abad-Mas, L. (2005). Memoria de trabajo en los procesos . básicos del aprendizaje. Revista de Neurología, 40 (supl.1).

9. Consultado el 22 de junio de 2008 en: http:// campusvirtual.uma.es/psicoev/Profesores/25. Romero/ Documentos/MT\%20y\%20aprendizaje.pdf

10. Fernández, E. A. (2001). . La Inteligencia Emocional y su relación con los Valores Interpersonales en los estudiantes del 5 to año de educación secundaria Tesis de Maestría, UNMSM, Lima
11. Gardner, H (1999) Las Inteligencias multiples estructura de la mente (3ra reimpresión) Colombia Nomos S.A.

12. Hernández Sampieri, R., Fernández Collado, C. \& Baptista Lucio, P. (2003). Metodología de la Investigación (3ra. Ed.). México: McGraw-Hill Interamericana..

13. Instituto Nacional de Estadística e Informática Inei (2009) Guía para la presentación de gráficos estadísticos Lima

14. Ministerio de Educación. (2004). Documento de trabajo UMC 11. La evaluación de la alfabetización lectora de PISA y el rendimiento de los estudiantes peruanos. Lima: UMC - MINEDU.

15. Mendoza C.A (2006). . La relación entre inteligencia emocional y rendimiento académico de los estudiantes de la Facultad de Ciencias Naturales y Matemáticas de la Universidad Nacional Federico Villarreal" Tesis de maestría UNFV

16. Mendoza C. A (2008). Inteligencias múltiples y rendimiento académico en los estudiantes de la Escuela Profesional de Matemática y Estadística de la Facultad de Ciencias Naturales y Matemática de la Universidad Nacional Federico Villarreal Tesis de Doctorado UNFV

17. Muños M. L (2008). Críticas de la evaluación censal de estudiantes 2007. Lima: UMC - MINEDU.

18. Munguía, U. (2003). . Relación entre la inteligencia emocional y las estrategias de aprendizaje como predictores del rendimiento académico en estudiantes universitarios Tesis de Maestría UNFV

19. Navarro, R (2003) El rendimiento académico; concepto, investigación y desarrollo España: Red Iberoamericana año/vol 1 número 002

20. Newman, J (1985) Sigma el mundo de la matemáticas. España: Grijalbo

21. Luca, S (2002) El docente y las inteligencias múltiples Buenos aires, Revista Iberoamericana de Educación 